Chapter 2

\title{
Functional Redundancy and Ecosystem Function - The Soil Microbiota as a Case Study
}

\author{
Stephanie D. Jurburg and Joana Falcão Salles \\ Additional information is available at the end of the chapter
}

http://dx.doi.org/10.5772/58981

\section{Introduction}

Understanding ecosystem functioning has been a main focus of ecological studies due to its importance for the maintenance of ecosystem integrity and human livelihood. While identifying and measuring relevant ecosystem functions may be a seemingly straightforward task, isolating the biota responsible for the provision of a particular function is far more complicated. In this context, understanding how biota influence ecosystem functioning remains a very active area of research in ecology, known as Biodiversity-Ecosystem Function (BEF) [1]. Given the accelerating rates of biodiversity loss [2] and predicted increases in the intensity and duration of extreme climate events [3], understanding how species interact to provide ecosystem functions is crucial for anticipating change as well as for establishing appropriate biodiversity buffers in order to minimize the risk of functional loss and maintain ecosystem integrity.

Functioning can be evaluated in the short-term, in which case the magnitude of the process is of interest, or in the long-term, measured as the probability that this is maintained in the face of environmental change. In both cases, functioning is an emergent property of ecosystems: interactions between the system's members and coevolution result in functioning which deviates from that expected from a system in which functioning was simply additive. In the case of environmental change, redundancy - the phenomenon in which a function is carried out by multiple species in an ecosystem - buffers functioning, as for any given environmental state there will be multiple organisms within a functional group which can perform optimally at a range of environmental conditions.

It has been suggested that concerns for the maintenance of biodiversity cannot be extended to microbes [4]. The implicit assumption is that microbial community composition is not relevant for determining function because microbes are endlessly diverse, so that the only filter 
determining their function is the environment. Specifically, in microbial systems, where diversity and abundance are extreme and growth rates are rapid, it was formerly assumed that redundancy is so high that diversity and community composition are decoupled from functioning due to the following observations: 1) most microbial species are ubiquitous and present in very low densities, awaiting an opportunity to "bloom"; 2) the rapid adaptability of microbes means that such a system will never be so impoverished as to cease functioning; and 3) the microbial system is so tightly linked to its physical environment that it cannot be studied within the context of cause-effect that is generally necessary for BEF studies. However, recent studies have shown that community composition matters to function $[5,6]$ : in soil, microbial communities exhibit a home-field advantage in decomposing endemic vs. foreign litter $[7,8]$ and different communities do not become more similar when exposed to the same environment [9]. This ongoing discussion has been particularly important in the realm of ecosystem models, where stable physical parameters or very coarse microbial parameters (such as total biomass) are assumed to accurately represent microbial contributions to ecosystem function [10].

Despite the current gaps in knowledge of microbial communities, this is an extremely attractive system for the study of BEF: the ease of manipulation, wide range of metabolic diversities, and availability of direct links between genetic diversity and function (i.e. functional gene analyses) allow for a range of experiments which would not be possible in other ecosystems. Particularly, the high turnover rate and diversity allow for studies which target the effect of redundancy on long-term function. A wide range of studies regarding this relationship are now available (for in-depth reviews, see [11,12]), but the results of microbial BEF studies have often been contradictory. The purpose of this chapter is to provide a comprehensive analysis of redundancy in microbial communities, paying special attention to the intricacies of these systems, in order to understand why these contradictions arise, and shed light on how redundancy might bolster ecosystem function in these extremely diverse ecosystems.

\section{Microbial diversity and its contribution to ecosystem function}

Microbial systems are responsible for the provision of a wide range of crucial ecosystem services, but little is known about the role of diversity in maintaining this function. This is mostly due to the overwhelming complexity found in them: the study of microbial communities has been likened to the study of solar systems [13]. This diversity is still not properly constrained: the lack of an ecological species definition for prokaryotes [14] has led to the usage of the operational taxonomic unit (OTU), defined as 97\% sequence similarity in the $16 \mathrm{~S}$ rRNA gene, is used as a threshold for prokaryotic species, however this threshold may not be comparable to the eukaryotic definition of species [14]. This means that most prokaryotes can be identified based on their sequences alone, which makes distinguishing rare species from sequencing errors nearly impossible [15], and obscures the definite measurement of prokaryotic diversity. Nevertheless, it is agreed that microbial diversity is extremely high: one gram of soil may contain $10^{3}-10^{6}$ unique taxa $[16,17]$. Furthermore, the link between phylogeny and function is truncated for prokaryotes, where horizontal gene transfer allows for the acquisition 
of functions - particularly those associated with adaptability to new environments - further complicates analyses of function through genes [18].

Despite these obstacles, microbial BEF-particularly for soil microbial communities demands much attention. In addition to serving as repositories of genetic information [19], they provide ecosystem services which are fundamental for human persistence, including the maintenance of agricultural systems and waste recycling [20]. In an assessment of the economic benefits of biodiversity, the soil microbiota was partly or fully responsible for waste recycling, soil formation, nitrogen fixation, bioremediation of chemicals, biotechnology, and biocontrol of pests. These services amounted to an estimated $\$ 1.16$ trillion dollars per year globally, which was over a third of the estimated annual contribution of terrestrial ecosystem services to the worldwide economy [21]. This study contrasts sharply with another estimate which, while considering both terrestrial and marine ecosystem services, differed in its estimate of the total annual value of these services by more than an order of magnitude[22]. This discrepancy illustrates the prevailing lack of consensus regarding the economic weight of ecosystem services, which is particularly problematic the face of biodiversity loss [20] because it obscures the value of preserving biodiversity for the sake of the services it provides. It also illustrates how functional classifications may be considered arbitrary: depending on the functions selected, how they are measured, and how they are valued, very different views of the same system can be obtained.

Novel technologies are beginning to open the door for the pursuit of deeper ecological understanding of microbial systems, but these advances are not accompanied by an increase in ecological theory. High-throughput sequencing has greatly accelerated the rate at which new microbial species can be detected, but their ecological properties remain a mystery [19]. Thus, although we know increasingly more about "who is there?", this information is not accompanied by characterization of the new species' niche spaces ("what are they doing?"), which precludes the understanding of how additional species affect function at an ecosystem level. Instead, the large majority of BEF studies in microbial ecology tend to focus on a single or few ecologically relevant functions, and often measure the abundance and diversity of functional groups or genes associated with those functions. For example, the soil microbiota play a crucial role in the nitrogen cycle and studies trying to understand the link between $\mathrm{N}$ associated functions and soil microbiota use functional genes associated with different steps of the cycle, such as those associated with nitrification and denitrification, as a way to cut through the overwhelming diversity found in soils, and focus on functionally relevant microbial community dynamics, which may scale up and affect functioning at the ecosystem level [23].

\section{Microbial BEF: A world of contradictions}

Due to their rapid generation times and the large diversity found in small volumes, microbial systems are ideal settings to probe BEF relationships, particularly in controlled laboratory microcosm experiments [19]. Indeed, while much remains unknown about the world's 
microbiota [24], microbial BEF research has seemingly kept pace with macroecological research [25]. The former, however, has been riddled with contradictory results, and evidence for a positive BEF relationship has not been as strong as for the latter. Some of these discrepancies may arise from the heterogeneity which is unique and inherent to the microbial system. From an environmental perspective, the extremely heterogeneous soil matrix may unevenly buffer the effect of environmental change, reducing the homogeneity of the community's response. It is also important to note that the phenomena occurring in microenvironments within which the soil microbiota exist are of necessity averaged out for measurement, as current methodologies require soil to be homogenized before studying [26]. Furthermore, while positive BEF relationships are expected [1], a negative relationship resulting from antagonistic interactions has been documented $[27,28]$.

Many contradictions have been attributed to differences in experimental setup. A recent metaanalysis indicates that most microbial BEF research has relied on comparative approaches, which test the BEF relationship across environmental gradients or treatments, rather than explicitly manipulating biodiversity [25] (Figure 1). The more common, comparative approaches are potentially riddled with hidden variables, and thus do not allow for the drawing of a direct link between diversity and function. For this reason, here we focus mainly on experiments which involve direct manipulation of diversity, which tend to find a strong, positive BEF relationship [13].

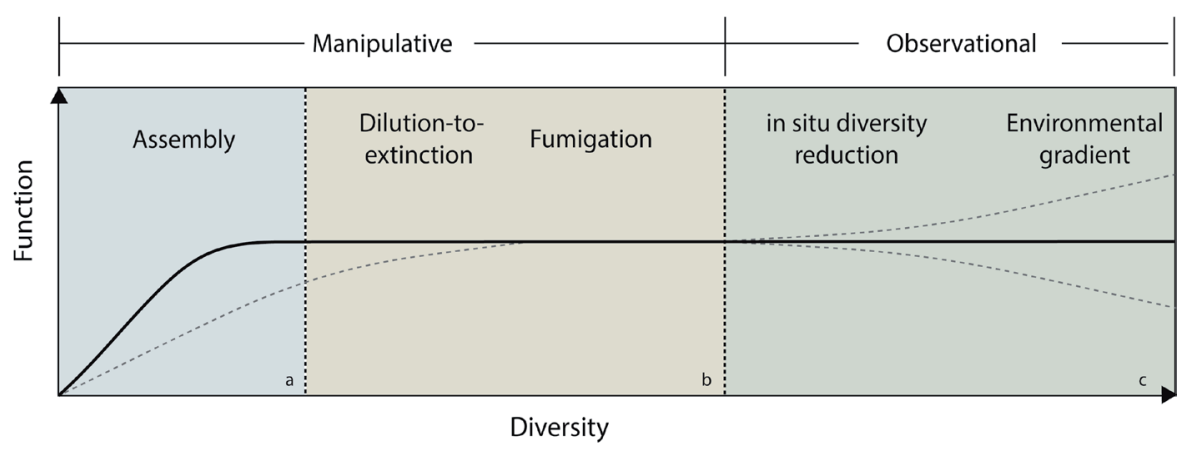

Figure 1. The relationship between diversity and function is asymptotic; different experimental approaches target different levels of species richness [13]. By greatly reducing diversity and environmental variability, assembly experiments seek mechanistic insight into the direct effect of diversity on process rates under minimized redundancy, that is, short-term function (a). Dilution-to-extinction and fumigation experiments retain greater species richness, and tend to emphasize the relationship between diversity and stability (i.e. long-term function) under otherwise stable environmental conditions (b). These experiments focus on systems in which the functioning asymptote is approached, although some dilution experiments cover broader ranges of diversity, as in [29] (b, dotted line). In observational studies, diversity is not manipulated, and the focus is rather on the effect of environmental change on the community's ability to maintain process rates (c). In this case, the level of redundancy is high enough to ensure no effect of diversity on functioning, although both positive and negative effects (c, dotted lines) have been observed for this type of experiments $[28,30]$ 
The manipulative experiments fit within two categories. In assembly experiments, a community is experimentally assembled to test the effect of each additional species or community structure on the community [31]. By studying overly-simplified communities, these studies tend to target the ecological functioning that arises from minimally redundant systems - that is, right before functioning begins to 'saturate' (Figure 1a). This approach has been criticized because it can only include culturable bacteria, which may represent less than $1 \%$ of soil microbes [32], and because the diversity levels achieved are always unrealistically low, and effects observed at such low diversity levels may not be relevant or applicable to more realistic scenarios and thus is not representative. Furthermore, this setup generally ignores the effect of historical selection patterns on community composition, which seems to be related to functioning as well [7]. Nevertheless, studying only culturable microbes allows for a full functional characterization of each population introduced into the system, and in this way over-yielding of the community as an emergent property of biodiversity can be studied mechanistically. For example, by characterizing 16 species of denitrifying bacteria in terms of their use of 6 carbon resources found in soil, Salles and colleagues created a model to predict $\mathrm{CO}_{2}$ production and denitrification based on the added functioning of each individual in the system. In this way, they were able to detect over-yielding and potential antagonism within their assembled communities [33]. This body of work has found a strong, positive BEF relationship, but has also stressed that it is the diversity of the functional traits in the community - not the number of taxa present - which affect functioning: for example, a recent 12strain assembly experiment found that the best predictor of function was the phylogenetic diversity of each microcosm [34], which agrees with previous findings [35]. The ability to manipulate genotypic and functional diversity as well as the distribution of species in assembled communities has been crucial for this [36,37]. Unfortunately, assembly experiments represent less than $1 \%$ of microbial BEF studies, and long-term studies using assembly experiments are non-existent: the lack of further mechanistic insight is one of the greater gaps in microbial BEF research $[13,25]$.

A second approach is to erode a large part of the microbial population selectively (e.g. using heat or chloroform) or randomly (reinocculating sterile soil with serial dilutions of the original community), in the so called removal experiments. These systems seem to maintain redundancy and a large part of their complexity, and much of the extant long-term BEF research has depended on removal microcosms (Figure $2 b$ ). The first studies on microbial BEF used these approaches [38], and together with subsequent works have found that broad microbial functions, such as organic matter decomposition, are not affected by large decreases in diversity, but that soils with lowered diversity seem to be less resistant to invasion and less resilient to disturbance [38,39]. Nevertheless, these studies have also yielded contradictory results. For example, in one case, microbial diversity was reduced by inoculating sterile soil with serial dilutions of its original community, but the rate of carbon mineralization, nitrification and denitrification enzyme activity were not related to the diversity treatments, even after diversity reductions of more than $99 \%$ of the soil biota, suggesting no BEF relationship [40]. Using the same serial dilution approach, another experiment found that while a $10^{-5}$ dilution led to a $75 \%$ decrease in estimated richness, the potential denitrification rates of these soils was reduced by about $75 \%$ as well, pointing at a strong, positive BEF relationship [29]. 
Soil microbes are intricately tied to their environment and to each other. The complexity of the system requires that it be simplified for study, but in doing so in ways which maintain an ecosystem which is representative of the natural one has been incredibly challenging [13]. The three approaches discussed here-comparative gradient analysis, assembly, and removal experiments - target the study of the effect of the environment, diversity, and redundancy on functioning, respectively.

\section{Functional redundancy and diversity}

Redundancy is a characteristic of ecological systems which arises when "different species perform the same functional role in ecosystems so that changes in species diversity do not affect ecosystem functioning", and must be defined relative to the system being studied [41]. The term was first developed in an attempt to optimize conservation efforts and direct them towards the most ecologically relevant species, highlighting the importance of diversity in maintaining functional stability and the integrity of the ecosystem in the face of environmental fluctuation [42], and was later taken up as a way to calculate how much biodiversity could be lost before it affected function [43].

Functional redundancy emerges from the functional classification of its individuals. In contrast to taxonomic classifications, functional ones group organisms based on their contribution to ecosystem functioning rather than phylogeny. This classification paradigm has several advantages: functional diversity is generally a better indicator of ecosystem functioning than the direct measurement of species richness [34,44-46], and functional classifications implicitly account for environmental and biotic interactions by measuring only the outcome of community composition, thereby overcoming the oversimplification which stems from studying individual species in a laboratory setting. While this classification scheme is not universally applicable in the sense that functions must be defined relative to the system, it allows for the comparison between ecosystems that contain different species [47].

A major obstacle in applying functional classifications is the different interpretations of what constitutes a functional group, functional guild, or functional type. While functional classifications are not new to ecology, they became popular fairly recently, with the definition of the functional guild as a conceptual tool: "...a group of species that exploit the same class of environmental resources in a similar way. This term groups together species, without regard to taxonomic position, that overlap significantly in their niche requirements. (...) A species can be a member of more than one guild" [48]. Since then, new terms (e.g. functional group, strategy, trait, etc.) emerged and were used to define slightly different, yet overlapping concepts (for an in-depth discussion, see [49]). While the concept was rapidly adopted by ecology, it was not applied rigorously during the development of classification schemes, rendering them incomparable in many cases. Perhaps the biggest problem has been differentiating between functional response groups (groups of organisms which respond similarly to changes in environmental factors) and functional effect groups (groups of organisms species which contribute in a similar way to ecosystem function) [50]. In order to understand the link 
between ecosystem functioning and biodiversity, both of these classifications are necessary: under a given environmental condition, knowing which organisms are in their optima and which are out of their functioning range precludes the understanding of how biodiversity affects function, as much of this diversity may be apparent in terms of functioning if the organisms are diverting resources from growth to persistence. Classifying organisms into functional response groups becomes even more important if the functions in question are longterm, and environmental variability is a factor (see section 5.2).

Nowhere is the need for functional effect classifications more important than in the soil microbiome, where it is estimated that $85 \%$ of microbial cells and over $50 \%$ of microbial OTU's are inactive at any given time [51]. This means that a majority of the soil microbial diversity is only apparent with regards to short term functioning (the long term implications of these 'microbial seed banks' are discussed in a later section). Despite the need, to our knowledge only one experiment has classified a set of microbes based on their response to environmental change [52]. In this study, respiration-which is related to growth-was used both as an indicator of function (functional effect trait) and fitness (functional response trait) for 23 individual strains of bacteria and 22 strains of fungi across a range soil moisture contents. While for some organisms the wettest soil coincided with the highest respiration, many strains exhibited optimal respiration rates at intermediate moisture contents. Different niche breadths - tolerance to a wide range of environmental change-were observed. There was a strong phylogenetic signal associated with moisture tolerance: closely related strains performed more similarly that would be expected if the relationship between phylogeny and functional response were random. Finally, it was observed that biofilm-producing organisms performed better at low moisture content and had a wider tolerance range, but grew more slowly, highlighting the fact that environmental adaptation requires trade-offs [52].

The above study created the first microbe-focused functional response classification, but did not further study whether these strains, when combined, behave similarly, or whether the behavior changes with increasing community diversity. To our knowledge, no such studies exist. The novel practice of seeking the 'core microbiome' of an environment-that is, to distinguish between microbial species which change in response to the environment [53]alludes to the need to group organisms based on their response traits, but it is generally measured in natural environments, and as such is riddled with confounding factors. One factor which distinguishes prokaryotes from other organisms is the ability to acquire mobile genetic elements (i.e. plasmids), which often contain genes that facilitate survival in a wider range of environmental states [18]. The potential change in response trait classification resulting from the acquisition of mobile genetic elements also remains unexplored.

\section{The additive effect of biodiversity}

The primary concern of BEF research is not the individual capacity of an organism to function, but rather the emergent properties that arise from biodiverse communities. This improvement in functioning may be an increase in functional output-known as the short term effects of 
biodiversity - or an increase in the probability that this level of functioning will be maintained given environmental change, known as the long term effects (Figure 2). These emergent properties are particularly hard to measure in complex systems due to the difficulty of partitioning and attributing changes in community function amongst a plethora of individuals.

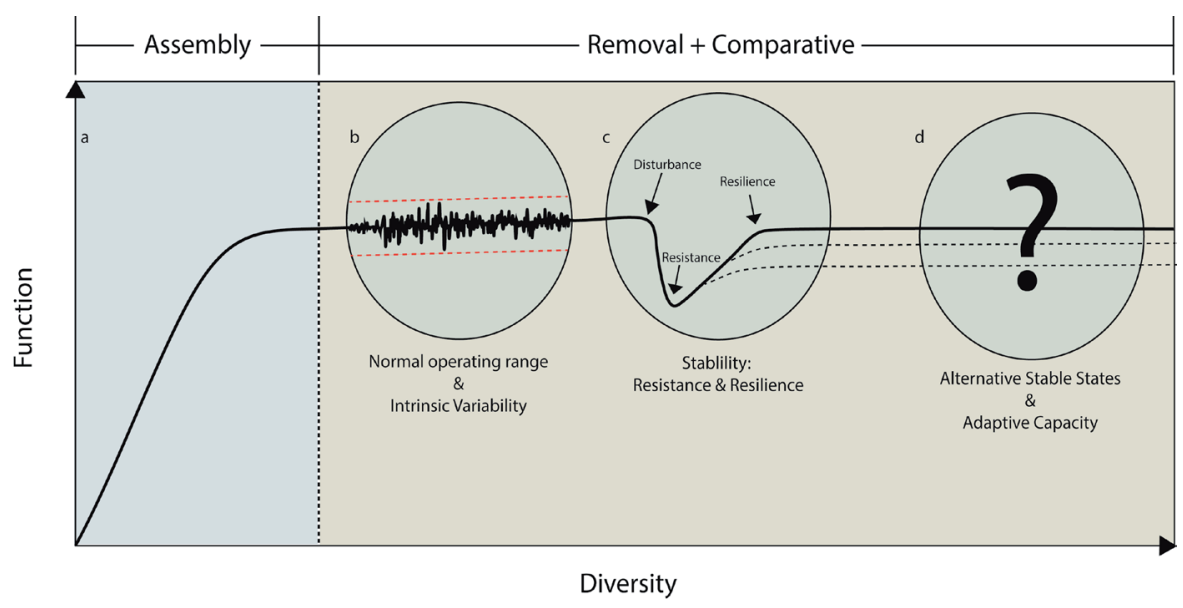

Figure 2. The short and long-term effects of biodiversity are studied in systems where diversity is simplified to different levels: for the former, the assembly approach discussed in section 3 is generally optimal (a), as simple systems are more tractable and it is easier to link an individual to increases in function. For the study of the long-term effects of biodiversity on ecosystem function - namely stability and adaptive capacity-more diversity is preserved. The emphasis is on monitoring the variability of functional parameters over time, if the goal is to determine intrinsic stability (b); or to measure resistance and resilience of the system to disturbance, if the focus is on functional stability sensu Pimm 1984 [54]. The study of alternative stable states and adaptive capacity is in its infancy, and even less is known regarding the redundancy on these two ecological properties in microbial systems.

\subsection{Short term effects: Productivity}

The idea that biodiversity increases ecosystem function was engraved in Darwin's original work "...if a plot of ground be sown with one species of grass, and a similar plot be sown with several distinct genera of grasses, a greater number of plants and dry herbage can be raised in the latter than in the former case (...) the truth of the principle that the greatest amount of life can be supported by the great diversification of life, is seen under many natural circumstances" [55]. At the most basic level, BEF research seeks to understand which characteristics arise from the presence of additional species in an ecosystem before ecosystem function begins to saturate (Figure 2a). These emergent properties-also known as biodiversity effects-are broadly categorized as selection or complementarity [25], and are considered to be the mechanistic processes by which more diverse ecosystems exhibit higher process rates.

Selection refers to the phenomenon in which a more diverse community will have a higher probability of containing more productive organisms. The better-performing organism tends 
to outcompete the rest for resources, returning the system to a monoculture in which its productivity dominates the entire system's productivity; interactions between competing species are not considered to be significant contributors to changes in function. Here, the maximum functioning for the community is determined by the rate of functioning of the most productive species $[25,56]$. In cases where the most competitive species is the less productive one, selection can lead to a negative BEF relationship.

Complementarity on the other hand, results from the competition for resources within a community, which may result in specialization and niche differentiation: as two species compete for a resource, they become specialized in exploiting the resource in different ways or times in order to minimize competitive pressure. In time, a greater efficiency is expected from the system as resources are used more thoroughly. Facilitation is a special case of complementarity, where mutualisms arise among organisms in a community, and result in higher ecosystem productivity [25]. While complementarity also predicts an asymptotic relationship between diversity and function, in this case the maximum productivity of the system may be higher than the productivity of any single member species-a phenomenon termed overyielding. In this scenario, the productivity of the system should be superior from the added productivities of the component species [57,58].

Evidence for resource-use complementarity in the soil microbial system is scant: in one case, microcosms containing up to 8 strains of cellulolytic bacteria were assembled and monitored over 25 days. Greater species richness supported more individuals and faster decomposition rates than any monoculture. Furthermore, the initial frequency distribution of inoculated organisms was maintained in the richest microcosms, suggesting coexistence, but it was not possible to distinguish whether this coexistence was due to niche differentiation or facilitation, although the authors suggest both mechanisms were present [59]. Similarly, in the assemblage experiment with denitrifying bacteria mentioned earlier, the expected function of an assembled community ('community niche') was calculated by summing the functioning of each of its members, and this was compared to the realized function. The most productive species in terms of $\mathrm{CO}_{2}$ did not coincide with the most productive denitrifiers, illustrating the danger of underestimating relevant species when a single function is used to study the community. In addition, community niche had a much greater explanatory power for the observed functions than species richness alone. The positive relationship between community niche and function suggested that the pattern of resource utilization of the species in a community are a major driver of the increased functioning resulting from higher diversity (i.e. complementarity). The authors also found a minor selection effect, where certain species had a greater effect on community functioning than others, but they argue that in such dynamic communities, teasing out the influence of selection from complementarity is irrelevant, as these are tightly intertwined [33]. In contrast, a study using a similar experimental approach found that respiration in assembled bacterial microcosms was lower in pairwise cultures than expected from the monocultures, and even lower in multispecies cultures, suggesting a predominance of negative interactions in this system [27]. 


\subsection{Long-term effects: Stability and resilience}

Ecosystems are dynamic, and communities must maintain ecological processes in the face of environmental change (stability), recover from radical environmental change (resilience), and adapt to constantly changing environments (self-organization) in order to persist. These three properties of diverse systems arise from the interplay between functionally redundant organisms in the community: species within a functional effect group might belong to different functional response groups. When environmental change occurs, it is the presence of organisms with different response patterns that allows for the maintenance of function, as species with more favorable responses to environmental change can compensate for the loss of function by the more sensitive species. In a similar way, the presence of functionally redundant organisms allows for other, tolerant individuals to maintain function when sensitive ones die or go dormant in response to disturbance.

Redundancy may be particularly important for the highly dynamic soil microbial system where, while diversity may be extreme, it may be necessary to buffer environmental change and guarantee the maintenance of function. The most well studied long-term BEF effect is functional stability. The notion that redundancy results in stability is not new, however interest in the development of mathematical models which mechanistically explain why this occurs did not become popular until the late 1990's. The importance of redundancy to ecosystem performance was initially modeled by applying concepts of reliability engineering to the stability of function [59]. In this model, ecosystem functioning was defined as "thebiogeochemical activities of an ecosystem or the flow of materials and processing of energy", complexity as the number of functional groups in the system, and reliability as the probability that the system will provide enough services to perpetuate the cycle. Here, diversity increases the stability within a functional group through compensatory growth, by which one species within a functional group increases when another is reduced. This refers to the difference in environmental tolerances between organisms, which suggests that in redundant systems, there is a higher probability that some organisms will be unaffected by the environmental change, and these will be able to use the resources left behind by the more sensitive species. Interestingly, this model looks at each functional group in the system as a compartment that feeds into the others, and so collapse of the system may come about if a single functional group becomes unstable.

The insurance hypothesis, developed a year later [60], builds on the previous model, and attributes the increase in functioning and decreased variability to the positive selection of the more productive species and the temporal asynchronicity of species responses to environmental fluctuation, respectively. Here, stability arises because the dynamics of the diverse systems are less dependent on individual species. This is particularly important in soils, which exhibit a very high species turnover rate: in one case, the bacterial and archaeal ammonia oxidizing communities in a range of Dutch agricultural soils showed above $50 \%$ change in community structure between seasons $[61,62]$. In another, it was shown that when colonizing a novel environment, the microbial community undergoes drastic rearrangement, and draws heavily from members of the 'rare biosphere' $[9,63]$, a strategy which may be crucial for stressresponse [51]. 
While the intrinsic variability of soils and the mechanisms that support it may be of interest to understanding how redundancy contributes to microbial ecosystem function (figure $2 b$ ), soil research rarely focuses on this aspect of stability. Instead soil stability is measured by applying a disturbance to soils with naturally or artificially differing levels of diversity and testing whether the microbiota are able to maintain function in the face of disturbance (resistance), and the time it takes the function to be restored to its pre-disturbance levels (engineering resilience, figure 2c) [54]. Redundancy can be measured as the diversity within a functional group, which is often assessed through functional gene markers that allow for the inclusion of unculturable organisms. As a whole, the results emerging from this area of research are hard to interpret: the usage of disturbances of different identity, duration, and intensity as well as the different time intervals between the measurements of resistance and resilience render these studies incomparable [64].

Nevertheless, this body of work has yielded important insights into the relationship between diversity and stability. For example, one study found that the diversity of both nitrite oxidizing and denitrifying bacteria in soil was not significant in determining the rate of functional recovery from experimental heating; rather, the main factor affecting this phenomenon was the abundance of the genes responsible for the functions tested [65]. In this case, it was not diversity, but sheer abundance which was responsible for stability. In another case, the recovery rate of two soils with naturally differing levels of diversity was compared: while mineralization of a labile carbon source $\left({ }^{14} \mathrm{C}\right.$-labeled wheat shoot) remained unaffected, mineralization of a recalcitrant substrate $\left({ }^{14} \mathrm{C}\right.$-labeled 2,4 dichlorophenol) was impaired. The more diverse soil was able to recover within the 9 weeks of the experiment, while the less diverse soil did not [30], suggesting here diversity mattered not only for stability, but also for the decrease in function.

Generally, narrower or less redundant functions have been found to be less stable following disturbance than broad functions[66], supporting the notion that biodiversity acts to buffer the system against fluctuations. In one case, respiration in serially diluted soil microbial microcosms exhibited no change in basal respiration or decomposition despite the large reductions in diversity, but nitrification was progressively retarded with each dilution [38]. Changes in community composition may affect function when, following disturbance, an abundant and efficient species is replaced by a redundant, but less efficient yet tolerant one. For example, monitoring potential nitrite oxidation (PNO) on soils that were treated with the cessation of tillage on tilled land or the establishment of tillage on untilled land, it was possible to detect a switch from Nitrobacter-like nitrite oxidizers to Nitrospira-like nitrite oxidizers with tillage, which explained the decrease in PNO [67]. The cessation of tillage did not result in a restoration of the Nitrobacter community within the 17 months of study, suggesting that long-term function might have been permanently affected by treatment. In an assembly experiment comparing the recovery from heating or metals in microbial communities of 1-12 bacterial species, biodiversity increased stability, measured as community biomass, but this stability was closely associated to the number of tolerant species in the community, a phenomenon analogous to the selection effect [34]. In a separate experiment, altering the $\mathrm{pH}$ in mixed culture fermentation reactors was shown to bring about the dominance of different species of Clostridium and elicit slight changes in the reactor's chemical output in accordance with the dominant species' preferences [68]. 
The distribution of species abundances within a community also affect stability: more evenly distributed communities are generally more stable than communities characterized by one or two dominant species $[37,69]$. In one case, the effect of selective stress on the stability of assembled denitrifying communities of up to 18 species was highly dependent on initial community evenness [37]. Even excluding the effect of the presence of tolerant species on the community's response, evenness played a significant role in maintaining stability.

Perhaps the clearest results have been obtained from studies looking at invasion resistance as an indicator of functional stability [11]. In general, diversity decreases invasibility in microbial systems $[36,39,45]$. By using both assembly and dilution in bacterial microcosms, a strong, negative correlation was shown between diversity and invasibility of an invader E. coli strain [39]. In a more recent experiment, the authors were able to attribute this decrease in invasibility to a reduction in easily available resources and reduced competitive advantage in the more diverse treatments. This result was confirmed by applying a resource pulse to the community following invasion, which led to an increase in the abundance of the invading species [70]. An analogous result was found in assembled communities consisting of different strains of Pseudomonas fluorescens, where genetic dissimilarity within a community increased productivity and decreased the success of the invader Serratia liquefaciens by decreasing the amount of resources available to the invader [71].

While it seems that theoretical predictions of a positive relationship between diversity and stability are somewhat in agreement, a large gap in the literature arises from differences between the definition of stability employed in these two fields: while experimental microbial ecology uses a functional definition of stability, which depends on resistance and resilience, theory often relies on intrinsic functional stability, which is a stand-alone parameter that measures the reduction of variability when there is no change in environmental parameters (Figure $2 b$ ). It is expected that more diverse communities will be more functionally stable and less compositionally stable, yet this has received little attention. The measurement of intrinsic stability requires the repeated measurement of an unperturbed community over time. Instead, a measurement is made immediately before disturbance to determine "normal" levels of functioning, immediately after to evaluate whether the system was resistant, and for a third time after a recovery period has passed. This approach does not consider that the system at equilibrium exhibits a constant variability which is intrinsic to the system, called normal operating range (NOR) [72], and thus cannot distinguish whether the response of a community to disturbance fits within 'normal' ranges of fluctuation or not, or whether a system that is deemed recovered is in a similar equilibrium to its undisturbed state.

\section{Moving forward: Redundancy and adaptive capacity}

The concept of resilience employed in the measurement of functional stability-engineering resilience-differs from the ecological concept as it was originally proposed [73]-ecological resilience. The former sees ecosystems as simple, rebounding springs, while the latter includes the possibility of the system shifting to alternative states due to perturbation, and thus is much harder to measure [73]. If ecological resilience is considered as a function in the same way as 
invasion resistance and stability, then it too can be progressively eroded. While research on this topic has been sparse and has not directly manipulated diversity, evidence of this phenomenon exists [74-78]. For example, mercury-contaminated, heat-shocked soils responded much more slowly to substrate additions than the transiently tylosin-contaminated or control soils [79]. The authors observed a significant decrease in the microbial diversity of the mercury-contaminated soils, which may explain the reduced response following additional disturbances. Mercury constitutes a long-term stress, so the heat-shocked communities were already coping with the original disturbance; however some studies find that even when the soils are allowed to recover from transient perturbations, their response to further disturbances is slower than that of the control soils: in another case, grassland soils which had experienced various forms of perturbation (reseeding, application of sewage-sludge, biocide/nitrogen and lime additions) recovered their ability to decompose more slowly following both copper and transient heat stresses than the unperturbed controls [78].

The concept of ecological resilience can be broken down into three characteristics: 1) the amount of change a system can undergo while retaining the same controls on function and structure; 2) the degree to which the system is capable of self-organization; and 3) the ability to build and increase the capacity for learning and adaptation [80]. Systems in which ecological resilience has been lost are unable to adapt to environmental change beyond a certain threshold, and in response to change shift to alternative stable states, in which the community is characterized by a different set of interactions (Figure 2d). One question that arises from this is whether microbial systems have stable equilibria to begin with. This is unclear, since the detection of alternative stable states requires the measurement of intrinsic variability which, as mentioned in the previous section, is not common practice in microbial ecology.

Another question is whether these irreversible shifts to alternative stable states have any relevance to ecosystem cycles. By analyzing the available literature, we may find mechanisms by which they do: the previously mentioned experiment in which tillage and no-tillage agricultural lands experienced an exchange in practice and the productivity and structure of the nitrite oxidizing communities underwent a catastrophic shift as, in response to an environmental change (tilling), the dominant members of a functional group (nitrite oxidizing bacteria) fundamentally changed from a those belonging to a more efficient genus (Nitrobacter) to a less efficient one (Nitrospira), leading to a decrease in function. Furthermore, cessation of tillage did not result in the opposite change in community. This may be an example of hysteresis - the phenomenon in which a system fails to return to its original state once perturbation ceases [81] - which is a property of systems that exhibit alternative stable states. In this case, the system may fail to return to its no-tillage state because the Nitrobacter community has been eroded beyond its ability to recover, or because the new dominant, generalist group is well suited for a wide range of environmental states, and it cannot easily be outcompeted when the system returns to its original conformation. Regardless of the underlying mechanism, this study provides evidence that a shift in the identity of the dominant organisms in a functional group may have an effect on functioning, and that this change may be irreversible. The implications of applying the ecological resilience concept to BEF studies are unknown but potentially very relevant, however to our knowledge, no work explicitly 
targets the measurement of ecological resilience in microbial systems, and this represents a serious gap in ecological research.

\section{Conclusion}

The last decade has seen a shift in focus, from a function-focused to a stability and probabilityfocused perspective. This is to be expected: at a time in which climate is expected to become more unpredictable [3], and biodiversity loss is expected to accelerate [2], it becomes important to be able to guarantee not only that ecosystems will be able to function, but that they will still be able to function in the face of drastic change. As mentioned earlier, the concept of functional redundancy was developed as a way determine which species within a community required the most conservation attention, and was later used to refer to a 'minimum' amount of biodiversity needed to keep the system functioning. As the focus shifts from the from the shortto the long-term effects of redundancy on ecosystem functioning, it becomes clear that the ecological value of redundant species lies in their ability to buffer against environmental change. Microbial communities are excellent model systems to study such buffering, not only due to the extremely high level of functional redundancy found here, but also due to the fact that these systems routinely experience rapid changes which may be catastrophic from a microbial perspective, and yet as a community they are able to maintain function. It seems that, even in the extremely diverse soil microbial system, diversity reductions result in reductions in either long-term or short-term function, or both, although the current gap in knowledge regarding microbial functional responses impairs our ability to understand the mechanics of this reduction as well as our ability to predict when environmental change results in functional change [82].

While the relevance of diversity to resilience and self-organization, and their contribution to the maintenance of function may be elusive and hard to study experimentally, these relationships warrant our attention. Initial studies have already revealed the importance of rare species in restructuring communities. Given current knowledge, it seems that in changing environments, every species matters, even in communities as diverse as the soil microbial community. Future research must delve into whether certain individuals matter more by evaluating the functional response profiles of individuals and communities, and quantifying the effect of changes of community composition on function.

\section{Author details}

Stephanie D. Jurburg and Joana Falcão Salles*

*Address all correspondence to: falcao.salles@rug.nl

Department of Microbial Ecology, Centre for Life Sciences, University of Groningen, Groningen, The Netherlands 


\section{References}

[1] Hooper D, Chapin F, Ewel J, Hector A, Inchausti P, Lavorel S, et al. Effects of biodiversity on ecosystem functioning: A consensus of current knowledge. Ecological monographs. 2005;75(1):3-35.

[2] Millenium Ecosystem Assessment. Ecosystems and human well-being: Synthesis. Ecosystems. Washington D.C.: Island Press; 2005.

[3] IPCC 2007. Climate change 2007: Synthesis report. Contribution of Working Groups I, II and III to the Fourth Assessment Report of the Intergovernmnetal Panel on Climate Change. II and III to the Fourth Assessment Report of the Intergovernmental Panel on Climate Change. Intergovernmental Panel on Climate Change, Geneva. Geneva: IPCC; 2007.

[4] Finlay BJ, Maberly SC, Cooper JI. Microbial diversity and ecosystem function. Oikos. 1997;80(2):209-13.

[5] Allison SD, Martiny JBH. Resistance, resilience, and redundancy in microbial communities. Proceedings of the National Academy of Science. 2008;105(Supplement 1): 11512.

[6] Reed HE, Martiny JBH. Testing the functional significance of microbial composition in natural communities. FEMS Microbiology Ecology. 2007 Nov;62(2):161-70.

[7] Keiser AD, Strickland MS, Fierer N, Bradford MA. The effect of resource history on the functioning of soil microbial communities is maintained across time. Biogeosciences. 2011 Feb 23;8(1):1643-67.

[8] Strickland MS, Lauber C, Fierer N, Bradford MA. Testing the functional significance of microbial community composition. Ecology. 2009 Feb;90(2):441-51.

[9] Pagaling E, Strathdee F, Spears BM, Cates ME, Allen RJ, Free A. Community history affects the predictability of microbial ecosystem development. The ISME journal. Nature Publishing Group; 2013;8(1):19-30.

[10] McGuire KL, Treseder KK. Microbial communities and their relevance for ecosystem models: Decomposition as a case study. Soil Biology and Biochemistry. 2010 Apr; 42(4):529-35.

[11] Shade A, Peter H, Allison SD, Baho DL, Berga M, Bürgmann H, et al. Fundamentals of Microbial Community Resistance and Resilience. Frontiers in Microbiology. 2012;3(December):1-19.

[12] Griffiths BS, Philippot L. Insights into the resistance and resilience of the soil microbial community. FEMS Microbiology Reviews. 2012;

[13] Le Roux X, Recous S, Attard E. Soil microbial diversity in grasslands and its importance for grassland functioning and services. In: Lemaire G, Hodgson J, Chabbi A, 
editors. Grassland Productivity and Ecosystem Services. Wallingford: CAB international; 2011. p. 158-65.

[14] Cohan FM. What are bacterial species? Annual Reviews in Microbiology. 2002;56(1): $457-87$.

[15] Pedrós-Alió C. The rare bacterial biosphere. Annual Review of Marine Science. Annual Reviews; 2012;4:449-66.

[16] Torsvik V, Øvreås L. Microbial diversity and function in soil: From genes to ecosystems. Current opinion in microbiology. 2002;5(3):240-5.

[17] Gans J, Wolinsky M, Dunbar J. Computational improvements reveal great bacterial diversity and high metal toxicity in soil. Science. 2005 Aug 26;309(5739):1387-90.

[18] Van Elsas JD, Jansson JK, Trevors JT, editors. Modern Soil Microbiology. 2nd ed. Boca Raton, Florida: CRC Press; 2006.

[19] Prosser JI, Bohannan BJM, Curtis TP, Ellis RJ, Firestone MK, Freckleton RP, et al. The role of ecological theory in microbial ecology. Nature Reviews Microbiology. 2007;5(5):384-92.

[20] Jeffery S, Gardi C, Jones A, Montanarella L, Marmo L, Miko L, et al. European Atlas of Soil Biodiversity. Luxembourg: Publications Office of the European Union; 2010.

[21] Pimentel D, Wilson C, McCullum C, Huang R, Dwen P, Flack J, et al. Economic and environmental benefits of biodiversity. BioScience. JSTOR; 1997;747-57.

[22] Costanza R, D'Arge R, De Groot R, Farber S, Grasso M, Hannon B, et al. The value of the world's ecosystem services and natural capital. Nature. Londres; 1997;387(6630): 253-60.

[23] Bouskill NJ, Tang J, Riley WJ, Brodie EL. Trait-based representation of biological nitrification: model development, testing, and predicted community composition. Frontiers in microbiology. 2012 Jan;3(October):364.

[24] Prosser JI. Ecosystem processes and interactions in a morass of diversity. FEMS Microbiology Ecology. 2012;

[25] Krause S, Le Roux X, Niklaus P a., Van Bodegom PM, Lennon JT, Bertilsson S, et al. Trait-based approaches for understanding microbial biodiversity and ecosystem functioning. Frontiers in Microbiology. 2014 May 27;5(May):1-10.

[26] Vos M, Wolf AB, Jennings SJ, Kowalchuk GA. Micro-scale determinants of bacterial diversity in soil. FEMS Microbiology Reviews. Wiley Online Library; 2013 Apr 3;119.

[27] Foster KR, Bell T. Competition, not cooperation, dominates interactions among culturable microbial species. Current Biology. Elsevier; 2012;22(19):1845-50. 
[28] Pereira e Silva M, Schlotter-Hai B, Poly F, Guillaumaud N, Schloter M, van Elsas J, et al. Phylogenetic distance and abundance of thaumarchaeal ammonia oxidizing communities affect potential nitrification rates in agricultural soils. submitted.

[29] Philippot L, Spor A, Hénault C, Bru D, Bizouard F, Jones CM, et al. Loss in microbial diversity affects nitrogen cycling in soil. The ISME journal. 2013 Aug;7(8):1609-19.

[30] Girvan MS, Campbell CD, Killham K, Prosser JI, Glover LA. Bacterial diversity promotes community stability and functional resilience after perturbation. Environmental Microbiology. 2005 Mar;7(3):301-13.

[31] Bell T, Newman JA, Silverman BW, Turner SL, Lilley AK. The contribution of species richness and composition to bacterial services. Nature. 2005;436(7054):1157-60.

[32] Torsvik V, Ovreas L, Thingstad TF. Prokaryotic diversity--magnitude, dynamics, and controlling factors. Science. 2002;296(5570):1064.

[33] Salles JF, Poly F, Schmid B, Le Roux X. Community niche predicts the functioning of denitrifying bacterial assemblages. Ecology. 2009 Dec;90(12):3324-32.

[34] Awasthi A, Singh M, Soni SK, Singh R, Kalra A. Biodiversity acts as insurance of productivity of bacterial communities under abiotic perturbations. The ISME journal. Nature Publishing Group; 2014;

[35] Salles JF, Le Roux X, Poly F. Relating phylogenetic and functional diversity among denitrifiers and quantifying their capacity to predict community functioning. Frontiers in microbiology. Frontiers Media SA; 2012;3.

[36] Jousset A, Schulz W, Scheu S, Eisenhauer N. Intraspecific genotypic richness and relatedness predict the invasibility of microbial communities. The ISME journal. Nature Publishing Group; 2011 Jul;5(7):1108-14.

[37] Wittebolle L, Marzorati M, Clement L, Balloi A, Daffonchio D, Heylen K, et al. Initial community evenness favours functionality under selective stress. Nature. 2009;458(7238):623-6.

[38] Griffiths B, Ritz K, Bardgett R, Cook R, Christensen S, Ekelund F, et al. Ecosystem response of pasture soil communities to fumigation-induced microbial diversity reductions: An examination of the biodiversity-ecosystem function relationship. Oikos. 2000;90(2):279-94.

[39] Van Elsas JD, Chiurazzi M, Mallon CA, Elhottovā D, Krištůfek V, Salles JF. Microbial diversity determines the invasion of soil by a bacterial pathogen. PNAS. National Acad Sciences; 2012;109(4):1159-64.

[40] Wertz S, Degrange V, Prosser JI, Poly F, Commeaux C, Freitag T, et al. Maintenance of soil functioning following erosion of microbial diversity. Environmental Microbiology. 2006;8(12):2162-9.

[41] Loreau M. Does functional redundancy exist? Oikos. 2004;3(140). 
[42] Walker BH. Biodiversity and ecological redundancy. Conservation Biology. 1992;6(1):18-23.

[43] Lawton JH, Brown VK. Redundancy in ecosystems. In: Schulze ED, Mooney HA, editors. Biodiversity and Ecosystem Function. Berlin, Heidelberg: Springer; 1994. p. 25570 .

[44] Petchey OL, Gaston KJ. Functional diversity (FD), species richness and community composition. Ecology Letters. 2002;5(3):402-11.

[45] Eisenhauer N, Schulz W, Scheu S, Jousset A. Niche dimensionality links biodiversity and invasibility of microbial communities. Functional Ecology. Wiley Online Library; 2013;27(1):282-8.

[46] Pereira e Silva Semenov, A.V., Schmitt, H, van Elsas, J.D., Falcao Salles, J. MC. Microbe-mediated processes as indicators to establish the normal operating range of soil functioning. Biology and Biochemistry. 2012;submitted.

[47] Voigt W, Perner J, Hefin Jones T. Using functional groups to investigate community response to environmental changes: Two grassland case studies. Global Change Biology. 2007 Aug;13(8):1710-21.

[48] Root RB. The Niche Exploitation Pattern of the Blue-Gray Gnatcatcher. Ecological Society of America. 1967;37(4):317-50.

[49] Gitay H, Noble IR. What are functional types and how should we seek them? In: Smith T, Shugart H, FI W, editors. Plant functional types: their relevance to ecosystem properties and global change. Cambridge University Press; 1997.

[50] Lavorel S, Garnier E. Predicting changes in community composition and ecosystem functioning from plant traits: revisiting the Holy Grail. Functional Ecology. Blackwell Science Ltd; 2002 Oct 1;16(5):545-56.

[51] Lennon JT, Jones SE. Microbial seed banks: the ecological and evolutionary implications of dormancy. Nature Reviews Microbiology. Nature Publishing Group; 2011;9(2):119-30.

[52] Lennon JT, Aanderud ZT, Lehmkuhl BK, Schoolmaster DR. Mapping the niche space of soil microorganisms using taxonomy and traits. Ecology. 2012 Aug;93(8):1867-79.

[53] Shade A, Handelsman J. Beyond the Venn diagram: the hunt for a core microbiome. Environmental Microbiology. 2012 Jan;14(1):4-12.

[54] Pimm SL. The complexity and stability of ecosystems. Nature. 1984;307(5949):321-6.

[55] Darwin C. On the origin of species by means of natural selection. Murray, London. 1859; 
[56] Tilman D, Lehman CL, Thomson KT. Plant diversity and ecosystem productivity: Theoretical considerations. Proceedings of the National Academy of Sciences. National Acad Sciences; 1997;94(5):1857-61.

[57] Tilman D, Lehman CL, Thomson KT. Plant diversity and ecosystem productivity: Theoretical considerations. Proceedings of the National Academy of Sciences. National Acad Sciences; 1997;94(5):1857-61.

[58] Loreau M. Biodiversity and ecosystem functioning: Recent theoretical advances. Oikos. 2000 Oct;91(1):3-17.

[59] Wohl DL, Arora S, Gladstone JR. Functional redundancy supports biodiversity and ecosystem function in a closed and constant environment. Ecology. 2004; 85(6): 1534-1540.

[60] Naeem S. Species redundancy and ecosystem reliability. Conservation Biology. 1998;12(1):39-45.

[61] Yachi S, Loreau M. Biodiversity and ecosystem productivity in a fluctuating environment: the insurance hypothesis. Proceedings of the National Academy of Sciences. 1999;96(4):1463.

[62] Pereira e Silva MC, Poly F, Guillaumaud N, van Elsas JD, Salles JF, Pereira E Silva MC. Fluctuations in ammonia oxidizing communities across agricultural soils are driven by soil structure and pH. Frontiers in Microbiology. 2012 Jan;3(March):77.

[63] Pereira e Silva MC, Dias ACF, van Elsas JD, Salles JF. Spatial and temporal variation of archaeal, bacterial and fungal communities in agricultural soils. PLOS ONE. Public Library of Science; 2012;7(12):e51554.

[64] Sjöstedt J, Koch-Schmidt P, Pontarp M, Canbäck B, Tunlid A, Lundberg P, et al. Recruitment of members from the rare biosphere of marine bacterioplankton communities after an environmental disturbance. Applied and Environmental Microbiology. Am Soc Microbiol; 2012;78(5):1361-9.

[65] Deng H. A review of diversity-stability relationship of soil microbial community: What do we not know? Journal of Environmental Sciences. The Research Centre for Eco-Environmental Sciences, Chinese Academy of Sciences; 2012 Jun;24(6):1027-35.

[66] Wertz S, Degrange V, Prosser JI, Poly F, Commeaux C, Guillaumaud N, et al. Decline of soil microbial diversity does not influence the resistance and resilience of key soil microbial functional groups following a model disturbance. Environmental Microbiology. 2007;9(9):2211-9.

[67] Balser TC, Firestone MK. Linking microbial community composition and soil processes in a California annual grassland and mixed-conifer forest. Biogeochemistry. 2005 Apr;73(2):395-415.

[68] Attard E, Poly F, Commeaux C, Laurent F, Terada A, Smets BF, et al. Shifts between Nitrospira-and Nitrobacter-like nitrite oxidizers underlie the response of soil poten- 
tial nitrite oxidation to changes in tillage practices. Environmental Microbiology. Wiley Online Library; 2010;12(2):315-26.

[69] Lu Y, Slater FR, Mohd-Zaki Z, Pratt S, Batstone DJ. Impact of operating history on mixed culture fermentation microbial ecology and product mixture. Water Science \& Technology. IWA Publishing; 2011;64(3):760-5.

[70] Degens BP, Schipper LA, Sparling GP, Duncan LC. Is the microbial community in a soil with reduced catabolic diversity less resistant to stress or disturbance? Soil Biology and Biochemistry. Elsevier; 2001;33(9):1143-53.

[71] Mallon C., Poly F, Le Roux X, Marring I, van Elsas J, Salles JF. Resource pulses can alleviate the biodiversity-invasion relationship in soil microbial communities. Ecology. (submitted).

[72] Jousset a, Schmid B, Scheu S, Eisenhauer N. Genotypic richness and dissimilarity opposingly affect ecosystem functioning. Ecology Letters. 2011 Jun;14(6):537-45.

[73] Kersting K. Normalized ecosystem strain: A system parameter for the analysis of toxic stress in (micro-) ecosystems. Ecological Bulletins. 1984;150-3.

[74] Holling CS. Resilience and stability of ecological systems. Annual Reviews in Ecology, Evolution and Systematics. 1973;1-23.

[75] Philippot L, Cregut M, Chèneby D, Bressan M, Dequiet S, Martin-Laurent F, et al. Effect of primary mild stresses on resilience and resistance of the nitrate reducer community to a subsequent severe stress. FEMS Microbiology Letters. 2008 Aug;285(1): $51-7$.

[76] Zhang B, Deng H, Wang H, Yin R, Hallett PD, Griffiths BS, et al. Does microbial habitat or community structure drive the functional stability of microbes to stresses following re-vegetation of a severely degraded soil? Soil Biology and Biochemistry. Elsevier Ltd; 2010 May;42(5):850-9.

[77] Tobor-Kaplon MA, Bloem J, De Ruiter PC. Functional stability of microbial communities from long-term stressed soils to additional disturbance. Environmental Toxicology and Chemistry. Wiley Online Library; 2006;25(8):1993-9.

[78] Chaer G, Fernandes M, Myrold D, Bottomley P. Comparative resistance and resilience of soil microbial communities and enzyme activities in adjacent native forest and agricultural soils. Microbial Ecology. Springer; 2009;58(2):414-24.

[79] Kuan HL, Fenwick C, Glover LA, Griffiths BS, Ritz K. Functional resilience of microbial communities from perturbed upland grassland soils to further persistent or transient stresses. Soil Biology and Biochemistry. 2006;38(8):2300-6.

[80] Müller AK, Westergaard K, Christensen S, Sørensen SJ. The diversity and function of soil microbial communities exposed to different disturbances. Microbial Ecology. Springer; 2002 Jul;44(1):49-58. 
[81] Carpenter SR, Brock WA. Adaptive capacity and traps. Ecology and Society. 2008;13(2):40.

[82] Beisner BE, Haydon DT, Cuddington K. Alternative stable states in ecology. Frontiers in Ecology and the Environment. Eco Soc America; 2003;1(7):376-82.

[83] Wallenstein MD, Hall EK. A trait-based framework for predicting when and where microbial adaptation to climate change will affect ecosystem functioning. Biogeochemistry. 2012;109(1):35-47. 
